\title{
Impact of Chronic Obstructive Pulmonary Disease on Patient with Acute Myocardial Infarction Undergoing Primary Percutaneous Coronary Intervention
}

\author{
Pei-Hsun Sung ${ }^{1}$, Sheng-Ying Chung ${ }^{1}$, Cheuk-Kwan Sun ${ }^{2}$, Cheng-Hsu Yang ${ }^{1}$, Shyh-Ming Chen ${ }^{1}$, \\ Chi-Ling Hang ${ }^{1}$, Chien-Jen Chen ${ }^{1}$, Kuo-Ho Yeh ${ }^{1}$, Yung-Lung Chen ${ }^{1}$, Chiung-Jen Wu ${ }^{1}$, \\ Hsuen-wen Chang ${ }^{3}$, Tzu-Hsien Tsai ${ }^{1}$, Hon-Kan Yip ${ }^{1}$
}

Background: This study reported the incidence and prognostic outcome of chronic obstructive lung disease (COPD) patients with acute ST-segment elevation myocardial infarction (STEMI) undergoing primary percutaneous coronary intervention (PCI).

Methods: Between January 2002 and May 2011, totally 1554 consecutive patients who experienced STEMI undergoing primary PCI were enrolled into the study.

Results: $\quad$ Of the 1554 patients, 124 (9.7\%) with diagnosis of COPD and $1430(90.3 \%)$ without COPD were categorized into group 1 and group 2. Although no difference in in-hospital mortality was noted between the two groups $(p=0.726)$. However, the hospitalization duration was notably longer $(p=0.003)$, the incidences of recurrent MI and re-hospitalization for congestive heart failure were significantly higher in group 1 than in group 2 (all $p<0.02$ ). Although Kaplan-Meier analysis demonstrated that the incidence of freedom from one-year major adverse clinical outcome (MACO) (defined as recurrent MI, re-admission for congestive heart failure was significantly lower in group 1 than group $2(p=0.012)$, multivariate Cox regression analysis showed COPD was not an independent predictor of MACO-free time after adjusting traditional risk factors.

Conclusion: COPD was not an independent predictor of short-term and medium-term MACO in patients with STEMI undergoing primary PCI. (Biomed J 2013;36:274-281)

\section{At a Glance Commentary}

Scientific background of the subject

Clinical observational studies have revealed that myocardial infarction and chronic obstructive pulmonary disease (COPD) tend to coexist and share similar prevalence. Recently, COPD has been further identified as an independent predictor of death after myocardial infarction. However, the majority of myocardial infarction patients in these studies did not receive primary percutaneous coronary intervention (PCI).

\section{What this study adds to the field}

This study investigated the incidence and prognostic outcome of COPD patients with acute ST-segment elevation myocardial infarction (STEMI) undergoing primary percutaneous coronary intervention. The results revealed that COPD was not an independent predictor of unfavorable clinical outcome in STEMI patients undergoing primary PCI.

Key words: acute myocardial infarction, chronic obstructive lung disease, clinical outcome

\footnotetext{
From the ${ }^{1}$ Division of Cardiology Division of Cardiology, Department of Internal Medicine, Kaohsiung Chang Gung Memorial Hospital and Chang Gung University College of Medicine, Kaohsiung, Taiwan, ${ }^{2}$ Department of Emergency Medicine, E-Da Hospital, I-Shou University, Kaohsiung, Taiwan, ${ }^{3}$ Department of Biological Sciences, National Sun Yat-Sen University, Kaohsiung, Taiwan Received: Feb. 01, 2012; Accepted: Nov. 15, 2012

Correspondence to: Dr. Hon-Kan Yip, Division of Cardiology, Department of Internal Medicine, Kaohsiung Chang Gung Memorial Hospital. 123, Dapi Rd., Niaosong, Kaohsiung 833, Taiwan (R.O.C.). Tel: 886-7-7317123 ext. 2363; Fax: 886-7-7322402; E-mail: han.gung@msa.hinet.net
}

DOI: $10.4103 / 2319-4170.113373$ 
$\mathrm{C}$ hronic obstructive pulmonary disease (COPD), which is characterized by poorly reversible airflow limitation, ${ }^{[1]}$ is a global epidemic affecting 5-15\% of all adults. ${ }^{[2]}$ Of importance is that COPD is the leading cause of both morbidities and mortality for hospitalized patients in various disease settings. ${ }^{[3-5]}$ Although COPD used to be considered a chronic inflammatory disease involving only the lung parenchyma, current evidence shows that it also systemically involves other organs and the circulation as reflected in the elevation of circulating inflammatory biomarkers, including cytokines and C-reactive protein that usually occurs in COPD patients. ${ }^{[6-8]}$ Accordingly, it is now realized that COPD is not only a heterogeneous disease with different disease activity, but its comorbid illnesses might also be major determinants of disease severity. ${ }^{[9]}$

Of all these comorbid diseases, cardiovascular, musculoskeletal, and psychological conditions have been reported to be the most prevalent. Of particular importance is that ischemic heart disease has been reported to be a leading cause of death in patients with COPD ${ }^{[10]}$ Indeed, the similarity between cardiovascular diseases and COPD are noteworthy. Not only has convincing evidence demonstrated a key role of endothelial dysfunction and atherosclerosis ${ }^{[11,12]}$ in the two disease entities, but they also share the common pathological contributors such as smoking and inflammation..$^{[7,13,14]}$ Besides, clinical observational studies have previously revealed that myocardial infarction and COPD tend to coexist and share similar prevalence in the population. ${ }^{[15,16]}$ Importantly, cardiovascular disease is the leading cause of death in COPD patients. ${ }^{[17,18]}$ Recently, some clinical observational studies ${ }^{[7,19-22]}$ have further identified COPD as an independent predictor of death after myocardial infarction.

However, the majority of myocardial infarction patients in these studies ${ }^{[7,19-22]}$ did not receive primary percutaneous coronary intervention (PCI) which has been well recognized as one of the best therapeutic strategies for reducing the short-term and long-term mortality in patients after ST-segment elevation myocardial infarction (STEMI). ${ }^{[23-25]}$

Thus, it is still unclear whether COPD has an independent impact on the outcome of patients with STEMI who underwent primary PCI or the outcome merely reflects the effect of smoking. Accordingly, this study, in addition to evaluating the incidence of COPD, tested the hypothesis that COPD might be an independent predictor for short-term and long-term untoward clinical outcome in STEMI patients undergoing primary $\mathrm{PCI}$.

\section{METHODS}

\section{Patient population and definition of chronic obstructive lung disease in study patients}

All patients with acute STEMI are considered eligible for primary PCI at our institute. Between January 2002 and
May 2011, 1,554 consecutive patients who experienced acute STEMI of $<12 \mathrm{~h}$ duration undergoing primary PCI were prospectively recruited in this study. All patients gave written informed consent for primary PCI in the emergency department. Our study is an observational study in COPD patients with STEMI undergoing primary PCI and approved by Institutional Reviewer Committee (Number: 100-3300B) at our institution.

\section{Definition and diagnosis of chronic obstructive lung disease}

COPD was defined according to one of the following criteria: (1) Information on COPD status was obtained by reviewing chart record of the need for pharmacologic therapy using bronchodilator agent; (2) Past history of a 1-second forced expiratory volume $<70 \%$ of the predicted value (by pulmonary function test) $;^{[1]}$ (3) Physical examination (by auscultation) showed expiratory wheezing and further confirmed by blood gas and chest radiograph (i.e., emphysematous change); or (4) Current use of bronchodilators prior to acute myocardial infarction (AMI). However, pulmonary function tests were not routinely performed at our institute to diagnose and assess the severity of COPD.

Of the 1,554 STEMI patients recruited in this study, $124(9.7 \%)$ who fitted the criteria of COPD were categorized into group 1 (i.e., COPD patients) with the remaining $1430(90.3 \%)$ without COPD being categorized into group 2.

\section{Procedure, protocol, and current medications}

Primary PCI was performed according to the protocol of our previous reports. ${ }^{[26-28]} \mathrm{A}$ transradial artery approach utilizing a 6-French arterial sheath is a routine procedure for acute STEMI at our institute unless Allen's test is positive on both sides. ${ }^{[29]}$ A 6-French Kimny guiding catheter (Boston Scientific, Scimed, Inc. Maple Grove, MN) was used for both the diagnosis of coronary artery occlusion and primary PCI. Intra-aortic balloon pump (IABP) support was introduced via right or left femoral arterial approach in patients experiencing acute pulmonary edema associated with unstable condition or hemodynamic instability.

All of our patients received a loading dose of clopidogrel (300 mg orally) in the emergency room, followed by a maintenance dose $(75 \mathrm{mg} /$ day orally once daily) for at least 3 months if bare metal stent deployment was performed or for at least 12 months if drug-eluting stent implantation was the chosen procedure. Aspirin (100 mg orally once daily) was given indefinitely to each patient. Other commonly prescribed medications also included 
angiotensin-converting enzyme inhibitors, angiotensin II type I inhibitors, statins, isonitrate, diuretics, and beta-blockers unless the presence of COPD with acute exacerbation or contra-indications for beta-blocking agents.

A loading dose of tirofiban ( $30 \mu \mathrm{g} / \mathrm{kg}$ of body weight) was administered to patients upon presentation at the emergency room, followed by a maintenance infusion of $0.15 \mu \mathrm{g} / \mathrm{kg} / \mathrm{min}$ for 18 to 24 hours at the beginning of this study. However, tirofiban therapy was subsequently withheld because it failed in providing any additional benefit for STEMI patients undergoing primary PCI. ${ }^{[26]}$ Therefore, only 268 (17.2\%) patients received tirofiban therapy in this study.

PercuSurge GuardWire was used when angiographic feature of high-burden thrombus formation was observed in the infarct-related artery. The indications and procedure protocol were described in details in our previous report. ${ }^{[27]}$

\section{Management strategy for obstructive non-infarct artery in study patients}

In the current study, three management strategies for multi-vessel disease were designed as described in our recent report. ${ }^{[28]}$ First, if non-infarct-related artery (non-IRA) had critical stenosis [defined as < (TIMI) grade 3 flow after thrombolysis in myocardial infarction, more than $90 \%$ stenosis, or evidence of plaque rupture with thrombus formation] associated with hemodynamic instability after patency of the IRA was resumed, one-stage treatment of both IRA and non-IRA was performed. Second, staged PCI was performed for non-IRA stenosis $75 \%$ after stabilization of the patient's condition during hospitalization or re-admission after uneventful discharge. Third, observation and 6-month angiographic follow-up was performed for a non-IRA stenosis $<70 \%$ and $>50 \%$.

\section{Definitions}

STEMI was defined as (1) typical chest pain lasting for more than 30 minutes with ST-segment elevation $>1 \mathrm{~mm}$ in two consecutive precordial or inferior leads, (2) typical chest pain lasting for more than 30 minutes with a new-onset complete left bundle branch block. Procedural success was defined as a reduction to residual stenosis of $<20 \%$ by balloon angioplasty or successful stent deployment at the desired position with a residual stenosis $<10 \%$ followed by TIMI grade 3 flow in the IRA. Multi-vessel disease was defined as stenoses of $50 \%$ in 2 major epicardial coronary arteries. Advanced congestive heart failure (CHF) was defined as New York Heart Association Functional Classification III. Major adverse clinical outcome (MACO) was defined as death, recurrent myocardial infarction, or readmission for CHF.

\section{Data collection}

The primary PCI program started at our institute since May 1993. For the purpose of this study, all patients undergoing primary PCI were prospectively recruited. Detailed in-hospital and follow-up data including age, gender, coronary risk factors, Killip score on admission, peak level of creatine phosphokinase (CPK), arrival time, duration from puncture to first balloon inflation, reperfusion time, duration of procedure, pre- and post-PCI TIMI flow grades, angiographic results, number of diseased vessels, in-hospital adverse events, and in-hospital mortality were obtained. These data were collected prospectively and entered into a digital database.

\section{Statistics}

Continue data which were expressed as mean \pm SD were compared using student $t$-test. Categorical data which were expressed as \% (n) were analyzed by $\chi^{2}$ test. Univariate Cox regression analysis was utilized to determine correlations between all baseline parameters and MACO-free time. Hazard ratio (HR) for MACO-free time was used using multiple Cox-regression analysis only parameters with a value of $p<0.1$ in univariate analysis were evaluated. Statistical analysis was performed using SPSS statistical software for Windows version 13 (SPSS for Windows, version 13; SPSS Inc., IL, U.S.A.). A $p<0.05$ was considered statistically significant.

\section{RESULTS}

\section{The baseline characteristics of study patients [Table 1]}

Patients in group 1 (i.e., with COPD) were significantly older than those in group 2 (i.e., without COPD). However, the gender, risk factors of coronary artery disease (CAD), and the incidences of previous stroke or old MI did not differ between the two groups. Electrocardiographic interpretation also showed no difference in infarction location between patients in group 1 and group 2. On the other hand, although the incidences of right ventricular infarction, Killip score 1, 2 or 4 did not differ between the two groups, the incidence of Killip score 3 was notably higher in group 1 than in group 2 patients. Furthermore, the peak level of CPK and CK-MB level were remarkably higher in group 1 patients than those in group 2. However, the white blood cell count and the estimated glomerular filtration rate were similar between the two groups.

The incidences of acute respiratory failure with requirement of mechanical ventilation, hemodynamic instability with requirement of intra-aortic balloon pump support, as well as the utilization of tirofiban and advanced 
Table 1: Baseline characteristics of study patients as seen in the study

\begin{tabular}{|c|c|c|c|}
\hline Variables & $\begin{array}{c}\operatorname{COPD}(+)^{*} \\
(n=124)\end{array}$ & $\begin{array}{c}\operatorname{COPD}(-)^{*} \\
(n=1430)\end{array}$ & $p$ value \\
\hline Age (years) & $68.5 \pm 9.9$ & $60.9 \pm 12.6$ & $<0.001$ \\
\hline Male gender & $85.5 \%(106)$ & $81.2 \%(1161)$ & 0.278 \\
\hline Hypertension & $54.8 \%(68)$ & $55.6 \%(795)$ & 0.925 \\
\hline Hypercholesterolemia & $39.5 \%(49)$ & $42.3 \%(605)$ & 0.571 \\
\hline Diabetes mellitus & $32.3 \%(40)$ & $36.2 \%(518)$ & 0.435 \\
\hline Current smoking & $37.1 \%(46)$ & $34.5 \%(493)$ & 0.556 \\
\hline Previous stroke & $8.9 \%(11)$ & $7.8 \%(111)$ & 0.604 \\
\hline Old myocardial infarction & $4.8 \%(6)$ & $4.4 \%(63)$ & 0.819 \\
\hline Infarction location by ECG & & & 0.707 \\
\hline Anterior wall infarction & $54.0 \%(67)$ & $55.9 \%(799)$ & \\
\hline Non-anterior wall infarction & $46.0 \%(57)$ & $44.1 \%(631)$ & \\
\hline Right ventricular infarction & $12.1 \%(15)$ & $10.5 \%(150)$ & 0.642 \\
\hline \multicolumn{4}{|l|}{ Killip score } \\
\hline Killip 1 & $53.2 \%(66)$ & $59.7 \%(853)$ & 0.182 \\
\hline Killip 2 & $19.4 \%(24)$ & $14.7 \%(210)$ & 0.189 \\
\hline Killip 3 & $16.1 \%(20)$ & $8.5 \%(121)$ & 0.008 \\
\hline Killip 4 & $11.3 \%(14)$ & $17.2 \%(246)$ & 0.103 \\
\hline WBC count $\left(\times 10^{3} / \mathrm{dL}\right)$ & $11.3 \pm 4.1$ & $11.1 \pm 3.9$ & 0.615 \\
\hline eGFR (ml/min) & $70.1 \pm 30.3$ & $65.6 \pm 27.3$ & 0.119 \\
\hline Peak CPK level (unit/L) & $3191 \pm 2997$ & $2665 \pm 2998$ & 0.007 \\
\hline Peak CK-MB level (unit/L) & $972 \pm 2561$ & $648 \pm 2137$ & 0.009 \\
\hline Acute respiratory failure & $12.1 \%(15)$ & $13.4 \%(192)$ & 0.783 \\
\hline $\begin{array}{l}\text { Intra-aortic balloon pump } \\
\text { support }\end{array}$ & $16.1 \%(20)$ & $20.7 \%(296)$ & 0.246 \\
\hline Tirofiban utilization & $21.0 \%(26)$ & $16.9 \%(242)$ & 0.265 \\
\hline $\operatorname{LVEF}(\%)$ & $54.3 \pm 16.3$ & $57.5 \pm 14.2$ & 0.039 \\
\hline Advanced $\mathrm{CHF}^{\star}$ & $25.0 \%(31)$ & $23.7 \%(339)$ & 0.742 \\
\hline
\end{tabular}

Continuous data were expressed as mean \pm SD, Categorical data were expressed as \% (n), Abbreviations: COPD: Chronic obstructive pulmonary disease; CK-MB: Creatine phosphokinase-MB; CPK: Creatinine phosphokinase; ECG: Electrocardiogram; eGFR: Estimated glomerular filtration rate; LVEF: Left ventricular ejection fraction; WBC: White blood cell count; *(+) indicated with COPD; (-) indicted without COPD; Indicated requirement of mechanical ventilatory support; ${ }^{*}$ Defined as New York Heart association functional Class III

CHF did not differ between the two groups. However, LVEF was notably lower in group 1 patients than those in group 2.

\section{Angiographic findings, current medications, short-term and medium-term clinical outcomes of study patients [Table 2]}

Angiographic studies demonstrated similar distribution of IRA between group 1 and group 2 patients. However, as compared with patients in group 2, those in group 1 had more severe obstruction of the IRA prior to primary PCI. There were no significant differences in the incidences of temporary pacemaker implantation due to heart block, stent implantation, utilization of PercuSurge wire for distal embolic protection, and successful reperfusion of the IRA.
Table 2: Angiographic results, current medications and clinical outcomes of study patients as seen in the study

\begin{tabular}{|c|c|c|c|}
\hline Variables & $\begin{array}{c}\operatorname{COPD}(+)^{*} \\
(n=124)\end{array}$ & $\begin{array}{c}\operatorname{COPD}(-)^{*} \\
(n=1430)\end{array}$ & $p$ value \\
\hline Multi-vessel disease & $59.7 \%(74)$ & $54.1 \%(774)$ & 0.259 \\
\hline \multicolumn{4}{|l|}{ Infarct-related artery } \\
\hline Left main trunk & $2.4 \%(3)$ & $3.2 \%(46)$ & 0.793 \\
\hline Left anterior descending artery & $50.0 \%(62)$ & $51.0 \%(729)$ & 0.852 \\
\hline Right coronary artery & $8.1 \%(10)$ & $8.6 \%(123)$ & 1.0 \\
\hline Left circumflex artery & $37.9 \%(47)$ & $36.5 \%(522)$ & 0.771 \\
\hline Saphenous vein graft & $1.6 \%(2)$ & $0.7 \%(10)$ & 0.248 \\
\hline Pre-PCI TIMI-flow grade & & & 0.036 \\
\hline$\geq 2$ & $19.4 \%(24)$ & $28.2 \%(403)$ & \\
\hline$\leq 1$ & $80.6 \%(100)$ & $71.8 \%(1027)$ & \\
\hline Pacemaker implantation & $6.5 \%(8)$ & $10.8 \%(154)$ & 0.166 \\
\hline Stent implantation & $83.9 \%(104)$ & $84.2 \%(1204)$ & 0.898 \\
\hline PercuSurge utilization & $36.3 \%(45)$ & $35.7 \%(510)$ & 0.922 \\
\hline Final TIMI flow & & & 0.333 \\
\hline TIMI-3 flow & $91.9 \%(114)$ & $94.0 \%(1344)$ & \\
\hline TIMI flow 2 & $7.1 \%(10)$ & $6.0 \%(86)$ & \\
\hline B-blocker utilization & $41.1 \%(51)$ & $62.5 \%(894)$ & $<0.001$ \\
\hline $\begin{array}{l}\text { Calcium channel blocker } \\
\text { utilization }\end{array}$ & $8.9 \%(11)$ & $5.0 \%(72)$ & 0.091 \\
\hline ACEI/ARB utilization & $75.8 \%(94)$ & $72.4 \%(1036)$ & 0.463 \\
\hline Statin utilization & $39.5 \%(49)$ & $42.8 \%(612)$ & 0.508 \\
\hline Duration of hospitalization (day) & $11.2 \pm 12.4$ & $8.1 \pm 11.2$ & 0.003 \\
\hline In-hospital mortality & $6.5 \%(8)$ & $7.9 \%(113)$ & 0.726 \\
\hline Stage PCI within one year & $18.5 \%(23)$ & $17.0 \%(243)$ & 0.621 \\
\hline Recurrent myocardial infarction & $4.8 \%(6)$ & $1.3 \%(19)$ & 0.011 \\
\hline Re-admission for $\mathrm{CHF}$ & $9.7 \%(12)$ & $2.2 \%(32)$ & $<0.001$ \\
\hline Cumulative mortality in one year & $14.5 \%(18)$ & $15.1 \%(216)$ & 1.0 \\
\hline One year $\mathrm{MACO}^{\dagger}$ & $28.2 \%(35)$ & $18.3 \%(262)$ & 0.012 \\
\hline
\end{tabular}

Continuous data were expressed as mean $\pm \mathrm{SD}$, Categorical data were expressed as \% (n), Abbreviations: ACEI/ARB: Angiotensin-converting enzyme inhibitors/Angiotensin II type I receptor blockers; CHF: Congestive heart failure; COPD: Chronic obstructive pulmonary disease; MI: Myocardial infarction; PCI: Percutaneous coronary intervention; TIMI: Thrombolysis in myocardial infarction; MACO: Major adverse clinical outcome; *(+): Indicated with COPD; $(-)$ : Indicted without COPD; 'Defined as death, recurrent myocardial infarction or re-admission for congestive heart failure

The incidences of current medical use, including calcium-channel blockers, angiotensin converting enzyme inhibitors/angiotensin II type I inhibitors and statins did not differ between the two groups. However, the incidence of $\beta$-blocker use was remarkably lower in group 1 than in group 2. Although in-hospital and one-year cumulative mortality rates did not differ between patients in the two groups, the duration of hospitalization was markedly increased in group 1 than in group 2. Additionally, the incidences of recurrent $\mathrm{MI}$ and readmission for $\mathrm{CHF}$ were significantly higher in group 1 than in group 2 patients. Furthermore, the one-year MACO was also significantly higher in group 1 than in group 2. 


\section{Univariate and multivariate cox regression} analysis of predictors for maco-free time

\section{[Tables 3 and 4]}

Univariate Cox regression analysis demonstrated that the baseline variables, including advanced age, female gender, and the presence of diabetes mellitus were strongly predictive of MACO-free time. Additionally, laboratory findings showed that white blood cell count and peak level of CPK were significantly associated with MACO-free time, whereas increased estimated glomerular filtration rate (eGFR) was significantly predictive of freedom from MACO-free time. Angiographic and electrocardiographic findings revealed that multi-vessel disease, failure in re-

Table 3: Univariate analysis* of predictors for major adverse clinical outcome free time

\begin{tabular}{lccc}
\hline Variables & HR & $95 \%$ CI & $p$ value \\
\hline Age & 1.056 & $1.044-1.068$ & $<0.001$ \\
Female gender & 2.289 & $1.635-2.932$ & $<0.001$ \\
Diabetes mellitus & 1.832 & $1.418-2.368$ & $<0.001$ \\
White blood cell count & 1.095 & $1.063-1.128$ & $<0.001$ \\
Peak level of creatine phosphokinase & 1.081 & $10.41-1.126$ & $<0.001$ \\
Increased eGFR & 0.971 & $0.966-0.976$ & $<0.001$ \\
Anterior wall MI & 1.473 & $1.135-1.912$ & 0.004 \\
Multi-vessel disease & 2.281 & $1.722-3.020$ & $<0.001$ \\
Failed reperfusion & 5.329 & $3.490-8.136$ & $<0.001$ \\
Increased LVEF & 0.954 & $0.944-0.965$ & $<0.001$ \\
Chronic obstructive pulmonary disease & 1.753 & $1.160-2.651$ & 0.008 \\
Advanced CHF & 5.871 & $4.471-7.710$ & $<0.001$ \\
\hline Abbreviati & &
\end{tabular}

Abbreviations: AWMI: Anterior wall myocardial infarction; eGFR: Estimated glomerular filtration rate; CHF: Congestive heart failure; CI: Confidence interval; LVEF: Left ventricular ejection fraction; HR: Hazard ratio; MACO: Major adverse clinical outcome; *All variables in Tables 1 and 2 were enrolled for univariate analysis by Cox regression and only those variables with $p<0.05$ were shown in the Table 3; 'Death, recurrent myocardial infarction or re-admission for congestive heart failure; Defined as CHF New York heart association functional Class III

Table 4: Multivariate cox regression analysis of predictors for major adverse clinical outcome free time*

\begin{tabular}{lccc}
\hline Variables & HR & $95 \%$ CI & $P$ value \\
\hline White blood cell count & 1.068 & $1.031-1.106$ & $<0.001$ \\
eGFR & 0.990 & $0.983-0.997$ & 0.003 \\
Age & 1.046 & $1.030-1.064$ & $<0.001$ \\
Diabetes mellitus & 1.557 & $1.117-2.172$ & 0.009 \\
Multiple vessel disease & 1.521 & $1.061-2.180$ & 0.022 \\
Peak level of CPK & 1.081 & $10.41-1.126$ & 0.018 \\
Left ventricular ejection fraction $^{\text {Advanced heart failure }}$ & 0.971 & $0.961-0.997$ & $<0.001$ \\
Adva $^{\dagger}$ & 1.925 & $1.356-2.734$ & $<0.001$ \\
\hline
\end{tabular}

Abbreviations: CI: Confidence interval; CKP: Creatinine phosphokinase; eGFR: Estimated glomerular filtration rate; HR: Hazard ratio; *: MACO: Major adverse clinical outcome: Defined as death, recurrent myocardial infarction or re-admission for congestive heart failure; 'Defined as congestive heart failure New York heart association functional Class III perfusion therapy, and anterior wall MI were significantly correlated with MACO-free time. Moreover, COPD and advanced CHF showed strong positive correlation with MACO-free time, whereas increased LVEF was significantly negatively predictive of MACO-free time. However, the MACO-free time of all patients did not differ between with and without utilizing $\beta$-blocker agent [HR: 0.796 (0.404-1.572), $p=0.23$ ]. Additionally, the COPD patients, the MACO-free time also did not differ between with and without using $\beta$-blocker agent $[29.4 \%(15 / 51)$ vs $27.4 \%$ (20/73), $p=0.851]$.

Multivariate Cox regression analysis demonstrated that age, white blood cell count, diabetes mellitus, peak level of CPK, and advanced CHF, but not COPD, were significantly and independently predictive of MACO-free time, whereas increased eGFR and LVEF were strongly and independently predictive of freedom from MACO-free time.

\section{Correlation between one-year cumulative maco-free survival and copd [Figure 1]}

The Kaplan-Meier analysis comparing the MACO-free survival rate of between patients with COPD and without COPD and demonstrating the one-year MACO-free survival rate was notably lower in patients with COPD than without COPD.

\section{DISCUSSION}

This study, which investigated the incidence of COPD in patients with STEMI undergoing primary PCI and the impact of COPD on the prognostic outcome of this patient population, yielded several striking implications. First, to the best of our knowledge, this is the first large-scale cohort

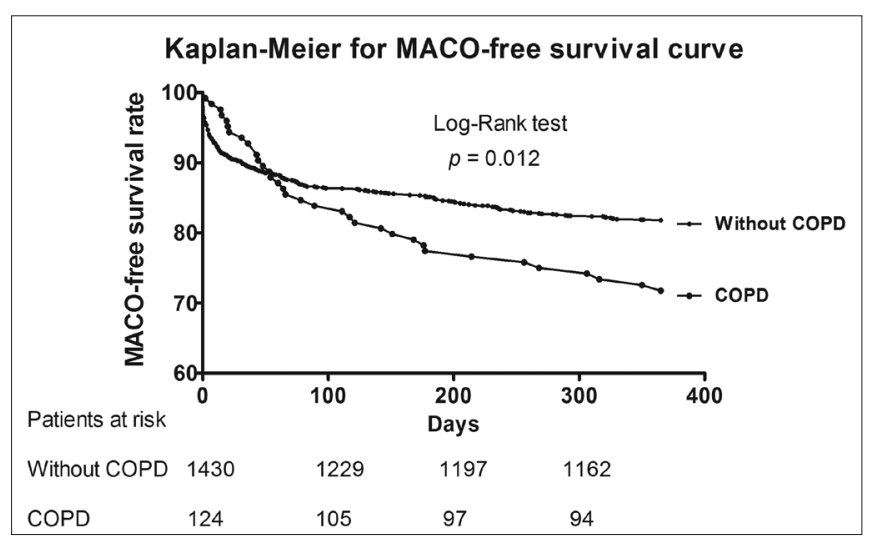

Figure 1: The kaplan-meier analysis comparing the MACO-free survival rate of between patients with COPD and without COPD and demonstrating the MACO-free time survival rate was notably lower in patients with COPD than without COPD $(p=0.012)$. Abbreviation used: MACO: Major adverse clinical outcome: Defined as death, recurrent myocardial infarction or re-admission for congestive heart failure.

Biomed J Vol. 36 No. 6

November - December 2013 
study investigating the outcome of COPD patients undergoing primary PCI for STEMI. Second, in the setting of AMI, the incidence of COPD in Chinese population was similar to that in the Western population. ${ }^{[7,19-22]}$ Third, in patients with STEMI undergoing primary PCI, COPD was not an independent predictor of short-term and medium-term mortality. Finally, COPD was insignificantly predictor of MACO-free time after adjusting traditional risk factors.

The results of previous studies ${ }^{[7,19-22]}$ have demonstrated that COPD was independently predictive of both short-term and long-term mortality. The major predictive factors of short term mortality in patients with AMI undergoing primary PCI were cardiogenic shock and unsuccessfully reperfusion. ${ }^{[25,28]}$ Surprisingly, the clinical presentation and the indexes of hemodynamic and respiratory instabilities such as acute respiratory failure, incidence of cardiogenic shock with requirement of intra-aortic balloon pump support did not show any significant difference between patients with and without COPD in the present study except for age, peak level of CPK, incidence of Killip 3 score, LVEF [Table 1], and the duration of hospitalization [Table 2]. Importantly, COPD was not an independent predictor of short-term or long-term mortality in the present study. Our findings, therefore, were inconsistent with those of the previous studies. [7,19-22] The inconsistent findings between the present study and the recent reports ${ }^{[7,19-22]}$ remain poorly explained. The following reasons may explain the discrepancy. ${ }^{[7,19-22]}$ First, the enrollment and exclusion criteria and the diagnosis of COPD may not be identical between our investigation and studies by others. ${ }^{[7,19-22]}$ The diagnosis of COPD was obtained from only hospital records and questioning the patient or only by 1 -second forced expiratory volume $<70 \%$ of the predicted value. Our patients with COPD was defined according to one of the following criteria: (1) Information on COPD status was obtained by reviewing chart record of the need for pharmacologic therapy using bronchodilator agent; (2) Past history of a 1-second forced expiratory volume $<70 \%$ of the predicted value (by pulmonary function test) ${ }^{[1]}$ (3) Physical examination (by auscultation) showed expiratory wheezing and further confirmed by blood gas and chest radiograph (i.e., emphysematous change); or (4) Current use of bronchodilators prior to acute myocardial infarction (AMI). Because the pulmonary function test could not be performed for everyone, we enrolled the patients with clinically suspicious COPD as far as possible.

Second, primary PCI, which was not a criterion for study enrollment in the majority of recent studies, ${ }^{[7,19-22]}$ was performed in all patients in the present study. Convincing data have established that primary PCI is one of the most effective life-saving procedures that also help in preserving heart function and reducing short-term and long-term mortality in patients after AMI. ${ }^{[23-25]}$ The results of the current study imply that successful primary PCI may abolish the independent influence of COPD on the clinical outcome of patients after AMI. Interestingly, a recent prospective, multicenter, multinational study of 8167 consecutive patients hospitalized for acute coronary syndrome in 6 Middle Eastern countries has demonstrated that COPD was not associated with increased risk of in-hospital mortality. ${ }^{[30]}$

The traditional risk factors shown in Table 4, which have been reported to be strongly and independently predictive of unfavorable clinical outcome, ${ }^{[12,25-27,31]}$ were found to be independently predictive of MACO-free time. Our findings, therefore, reinforced those of previous studies. ${ }^{[12,25-27,31]}$ In the current study, although univariate analysis showed that COPD was strongly associated with MACO-free time [Table 3 and Figure 1], multivariate Cox regression analysis did not produce consistent result. These findings suggest that, compared with traditional-risk factors in Table 4, COPD was a weaker predictor of clinical outcome in STEMI patients undergoing primary PCI.

COPD and CAD are frequently found to share common risk factors such as smoking and inflammation. ${ }^{[7,13,14]}$ This could explain the prevalence of these two disease entities in patients with AMI. ${ }^{[15,16]}$ In the present study, we found that the incidence of COPD in STEMI patient undergoing primary PCI was $9.7 \%$. Previous studies have shown that the incidence of COPD in Western AMI patients was around $8.6 \%$ to $12.0 \% .^{[7,19-22]}$ Thus, the incidence of coexistence of COPD and AMI was similar between the oriental and Western populations regardless of the ethnical difference.

Interestingly, previous studies have demonstrated that, as compared with AMI patients without COPD, those with COPD have significantly higher incidence of CAD risk factors, more deteriorated renal function, more unstable clinical presentations, and higher prevalence of hemodynamic instability and cardiogenic shock, as well as higher in-hospital mortality and long-term mortality rate. ${ }^{[7,19-22]}$

Of particular importance is that our study showed that COPD was strongly associated with recurrent $\mathrm{MI}$ and readmission for CHF. The patients with COPD were associated with older age, higher peak level of CPK, higher incidence of Killip 3 score, worsen LVEF. These findings may explain COPD was strongly associated with recurrent MI and readmission for CHF. Previous clinical observational study has revealed that COPD was associated with increased risk of CHF. ${ }^{[30]}$ In this way, our finding was consistent with the result of that study. ${ }^{[30]}$

\section{Study limitations}

This study has limitations. First, pulmonary function tests were not routinely performed to diagnose and assess the severity of COPD in the study patients during hospitalization. Thus, the incidence of COPD in patients with STEMI undergoing primary PCI may be underestimated 
in the current study. Second, since some patients died of cardiogenic causes within a few hours after the primary PCI procedure, the diagnosis of COPD usually could not be made in those patients.

\section{CONCLUSIONS}

COPD was no longer an independent predictor of short-term and medium-term MACO for patients with STEMI undergoing primary PCI.

\section{REFERENCES}

1. Stanojevic S, Wade A, Stocks J, Hankinson J, Coates AL, Pan H, et al. Reference ranges for spirometry across all ages: A new approach. Am J Respir Crit Care Med 2008;177:253-60.

2. Pauwels RA, Buist AS, Calverley PM, Jenkins CR, Hurd SS. Global strategy for the diagnosis, management, and prevention of chronic obstructive pulmonary disease. Nhlbi/who global initiative for chronic obstructive lung disease (gold) workshop summary. Am J Respir Crit Care Med 2001;163:1256-76.

3. Vestbo J, TORCH Study Group. The TORCH (towards a revolution in COPD health) survival study protocol. Eur Respir J 2004;24:206-10.

4. Yawna BP, Kaplanc A. Co-morbidities in people with COPD: A result of multiple diseases, or multiple manifestations of smoking and reactive inflammation? Prim Care Respir J 2008;17:199-205.

5. Bursi F, Vassallo R, Weston SA, Killian JM, Roger VL. Chronic obstructive pulmonary disease after myocardial infarction in the community. Am Heart J 2010;160:95-101.

6. Sin DD, Man SF. Systemic inflammation and mortality in chronic obstructive pulmonary disease. Can J Physiol Pharmacol 2007;85:141-7.

7. Dahl M, Vestbo J, Lange P, Bojesen SE, Tybjaerg-Hansen A, Nordestgaard BG. C-reactive protein as a predictor of prognosis in chronic obstructive pulmonary disease. Am J Respir Crit Care Med $2007 ; 175: 250-5$

8. Magnussen $\mathrm{H}$, Watz H. Systemic inflammation in chronic obstructive pulmonary disease and asthma: Relation with comorbidities. Proc Am Thorac Soc 2009;6:648-51.

9. Rabe KF, Wedzicha JA. Controversies in treatment of chronic obstructive pulmonary disease. Lancet 2011;378:1038-47.

10. Patel AR, Hurst JR. Extrapulmonary comorbidities in chronic obstructive pulmonary disease: State of the art. Expert Rev Respir Med 2011;5:647-62.

11. Ross R. Atherosclerosis: An inflammatory disease. N Engl J Med 1999;340:115-26.

12. Yip HK, Sun CK, Chang LT, Wu CJ. Strong correlation between serum levels of inflammatory mediators and their distribution in infarct-related coronary artery. Circ J 2006;70:838-45.

13. Sin DD, Man SF. Why are patients with chronic obstructive pulmonary disease at increased risk of cardiovascular diseases? The potential role of systemic inflammation in chronic obstructive pulmonary disease. Circulation 2003;107:1514-9.

14. Fabbri LM, Rabe KF. From COPD to chronic systemic inflammatory syndrome? Lancet 2007;370:797-9.
15. Barnes PJ. Chronic obstructive pulmonary disease. N Engl J Med 2000;343:269-80.

16. Pauwels RA, Buist AS, Calverley PM, Jenkins CR, Hurd SS. Global strategy for the diagnosis, management, and prevention of chronic obstructive pulmonary disease. NHLBI/WHO Global Initiative for Chronic Obstructive Lung Disease (GOLD) Workshop summary. Am J Respir Crit Care Med 2001;163:1256-76.

17. Anthonisen NR, Connett JE, Kiley JP, Altose MD, Bailey WC, Buist AS, et al. the Lung Health Study Research Group. Effects of smoking intervention and the use of an inhaled anticholinergic bronchodilator on the rate of decline of fev1. The lung health study. JAMA 1994;272:1497-505.

18. Camilli AE, Robbins DR, Lebowitz MD. Death certificate reporting of confirmed airways obstructive disease. Am J Epidemiol 1991;133:795-800.

19. Kjoller E, Kober L, Iversen K, Torp-Pedersen C. Importance of chronic obstructive pulmonary disease for prognosis and diagnosis of congestive heart failure in patients with acute myocardial infarction. Eur J Heart Fail 2004;6:71-7.

20. Salisbury AC, Reid KJ, Spertus JA. Impact of chronic obstructive pulmonary disease on post-myocardial infarction outcomes. Am J Cardiol 2007;99:636-41.

21. Hawkins NM, Huang Z, Pieper KS, Solomon SD, Kober L, Velazquez EJ, et al. Chronic obstructive pulmonary disease is an independent predictor of death but not atherosclerotic events in patients with myocardial infarction: Analysis of the valsartan in acute myocardial infarction trial (valiant). Eur J Heart Fail 2009;11:292-8.

22. Wakabayashi K, Gonzalez MA, Delhaye C, Ben-Dor I, Maluenda G, Collins SD, et al. Impact of chronic obstructive pulmonary disease on acute-phase outcome of myocardial infarction. Am J Cardiol 2010;106:305-9.

23. Grines CL, Browne KF, Marco J, Rothbaum D, Stone GW, $\mathrm{O}$ 'Keefe $\mathrm{J}$, et al. A comparison of immediate angioplasty with thrombolytic therapy for acute myocardial infarction. The primary angioplasty in myocardial infarction study group. N Engl J Med $1993 ; 328: 673-9$

24. Stone GW, Grines CL, Browne KF, Marco J, Rothbaum D, O'Keefe J, et al. Predictors of in-hospital and 6-month outcome after acute myocardial infarction in the reperfusion era: The primary angioplasty in myocardial infarction (pami) trail. J Am Coll Cardiol 1995;25:370-7.

25. Sheu JJ, Tsai TH, Lee FY, Fang HY, Sun CK, Leu S, et al. Early extracorporeal membrane oxygenator-assisted primary percutaneous coronary intervention improved 30-day clinical outcomes in patients with st-segment elevation myocardial infarction complicated with profound cardiogenic shock. Crit Care Med 2010;38:1810-7.

26. Yip HK, Wu CJ, Chang HW, Hsieh YK, Fang CY, Chen SM, et al. Impact of tirofiban on angiographic morphologic features of high-burden thrombus formation during direct percutaneous coronary intervention and short-term outcomes. Chest 2003;124:962-8.

27. Yip HK, Wu CJ, Chang HW, Hsieh YK, Fang CY, Chen SM, et al. Impact of tirofiban on angiographic morphologic features of high-burden thrombus formation during direct percutaneous coronary intervention and short-term outcomes. Chest 2003;124:962-8.

28. Chung SY, Lin FC, Chua S, Fu M, Wu CJ, Yip HK, et al. Clinical profile and outcome of first acute myocardial infarction with ischemic mitral regurgitation. Chang Gung Med J 2008;31:268-75.

29. Yang CH, Guo GB, Chen SM, Yip HK, Hsieh K, Fang CY, et al.

Biomed J Vol. 36 No. 6

November - December 2013 
Feasibility and safety of a transradial approach in intervention for chronic total occlusion of coronary arteries: A single-center experience. Chang Gung Med J 2010;33:639-45.

30. Hadi HA, Zubaid M, Al Mahmeed W, El-Menyar AA, Ridha M, Alsheikh-Ali AA, et al. Prevalence and prognosis of chronic obstructive pulmonary disease among 8167 middle eastern patients with acute coronary syndrome. Clin Cardiol 2010;33:228-35.

31. Tsai TH, Chai HT, Sun CK, Leu S, Fan CQ, Zhang ZH, et al. Comparison of 30-day mortality between anterior-wall versus inferior-wall st-segment elevation myocardial infarction complicated by cardiogenic shock in patients undergoing primary coronary angioplasty. Cardiology 2010;116:144-50. 